\section{Estado y empresarios en Corea del Sur: entre la reciprocidad y la autonomía}

La relación Estado-empresarios ha sido parte esencial en el estudio de las características propias del proceso de desarrollo socioeconómico en Corea del Sur. Los debates se han centrado en forma natural en el peso de los grandes conglomerados -o chaebols-, y en su capacidad para lograr

La relación Estado-empresarios ha sido parte esencial en el estudio de las características del proceso de desarrollo socioeconómico en Corea del Sur. Los debates se han centrado en el peso de los grandes

conglomerados y en su capacidad para lograr autonomía del Estado, en ocasiones en una postura binaria: control o no control, autonomía o no autonomía. En este

trabajo se pretende, con un enfoque de largo plazo, analizar las transformaciones en las relaciones Estado-empresarios en Corea y superar esta postura binaria.

Palabras clave: Estado, empresarios, política económica, Corea del Sur, Este de Asia.
- Profesor-investigador del Centro de Investigación Observatorio Social DESMOS, de la Universidad de Guadalajara. enrivalo@gmail.com autonomía del Estado, en ocasiones en una postura binaria: control o no control, autonomía o no autonomía, con el control estatal como parte del inicio de la industrialización coreana y la autonomía empresarial como resultado final del desarrollo tecnológico de los conglomerados. Los procesos históricos han sido más complejos y han generado una diversidad analítica destacable que supera con mucho los enfoques binarios fuertemente presentes: Amsden (1989) habla de "reciprocidad", ${ }^{1}$ Sakong (1993) de "reciprocidad incompleta", ${ }^{2}$ Weiss

\footnotetext{
I. Subsidios públicos a cambio de logros productivos y en el comercio internacional. En esta relación, el Estado juega el rol de "disciplinador".

2. Incompleta porque el apoyo público puede eliminarse a causa de un mal desempeño o no seguimiento de las directivas gubernamentales; porque implica la posibilidad del castigo.
} 


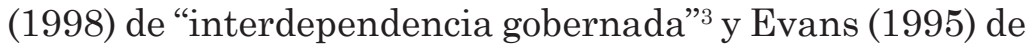
"autonomía incrustada". ${ }^{4}$ En este trabajo, intentamos con un enfoque de largo plazo analizar las transformaciones en las relaciones Estado-empresarios en Corea y su relación con el proceso de desarrollo económico, en diálogo con estos enfoques conceptuales.

\section{La reciprocidad incompleta y la amenaza}

Y el comienzo fue... el pecado: en 1961, la Junta dirigida por Park emprendió una campaña "anti-corrupción” contra las fortunas atesoradas "ilegalmente". ${ }^{5}$ Park Chung-Hee convocó a diez líderes, elegidos entre los más importantes empresarios, y logró con ellos un acuerdo, un "compromiso histórico" (Woo, 1991, p. 84): a cambio del retiro de la acusación criminal y del respeto a sus propiedades, los hombres de negocios iniciarían proyectos industriales deseados por las autoridades y darían una parte de ellos al gobierno. La

3. Relación institucionalizada de negociación Estado-empresarios. El Estado induce la cooperación empresarial y disciplina, requerimientos de desempeño económico de las empresas (citado en Kim, 2012B).

4. Es la sinergia entre Estado y sociedad, a través de redes densas de relaciones que conectan al Estado con las elites industriales (Evans, 2008). Los roles que el Estado puede desempeñar son "custodio" (establecimiento de reglas) o "demirugo" (generador de empresas públicas), "partero" (midwifery, apoyo a la emergencia de nuevo grupos o empresas) o "cuidador" (husbandry, apoyo y asistencia a las empresas a través de organizaciones públicas que toman tareas complementarias riesgosas, por ejemplo en ciencia y tecnología) (Evans, 1995, pp. I3-14).

5. La Junta arrestó a varios hombres de negocios por los delitos siguientes: transferencias ilegales de riquezas al extranjero, evasiones de impuestos hasta por 200 millones de hwans -la moneda de aquella época- o más, ganancias ilegales de más de 100 millones de hwans por la compra o por la renta de propiedades, donación de fondos políticos por más de 50 millones de hwans a cambio de créditos bancarios, y otros delitos. Estas definiciones de fortunas ilícitas provocaron que la mayor parte de los empresarios pudieran ser enjuiciados (Woo, 1991, p. 83). Lanzarotti (I992 A, p. 238) habla de este acontecimiento como del "singular episodio de la cacería de las fortunas ilegítimas". Lim (1991, p. 346) señala que el Estado coreano se manifiesta, de esta manera, como un productor de reglas, en este caso contra la corrupción y mucho más: reglas del juego para la relación empresarios-Estado, como veremos. 
Federación de Industrias Coreanas, organización representante de los intereses de los chaebols y creada en este contexto, sometió una serie de propuestas de inversión en industrias consideradas como prioritarias (Haggard y Moon, 1993, p. 66). El Estado así primero dirigirá y amenazará, después dirigirá y apoyará. El Estado y los capitales más importantes concluirán un compromiso histórico por la industrialización del país, bajo la amenaza y la restricción. Este acuerdo marcará las relaciones entre el Estado y el capital hasta nuestros días.

Esta relación singular provocará análisis diferentes, entre los que se distinguen los de Sakong (1993; ver también Jones y Sakong, 1980). Para Amsden (1989), en el modelo de acumulación coreano existe una reciprocidad entre el Estado y el sector privado:

En el corazón del modelo, había (a principios de los años 60) subsidios ofrecidos por el Estado a las empresas privadas a cambio de una producción más grande de exportaciones y de sustitutos de importaciones (pp. 63-145).

De esta manera, el Estado, para Amsden, juega un rol "disciplinario" no sólo frente al trabajo sino también ante el capital, a diferencia del paradigma del mercado en el que este rol es jugado por la mano invisible o del paradigma shumpeteriano en el que este rol pertenece al cambio tecnológico.

El carácter de reciprocidad implica, en general, un intercambio de la misma naturaleza. Sin embargo, la reciprocidad entre el Estado coreano y el sector privado no tenía este carácter, como la misma Amsden lo muestra, y era desigual. Además del intercambio de contrapartidas, el compromiso histórico de Park había marcado esta relación desde el ángulo de la amenaza. A causa de las acciones fuertemente desalentadoras y amenazadoras por parte del poder público, 
las empresas coreanas comprendieron rápidamente que la mejor manera de sobrevivir y de prosperar era "cumplir las directivas del gobierno" (Song, 1990, p. 145). ${ }^{6}$

Esta actitud fue analizada por Lanzarotti (1992, p. 238) como la "interiorización por los jefes de empresa de la coerción estatal"; los empresarios se sentían "respaldados" (reciprocidad) pero también "amenazados". Esta interiorización se convierte en habitus, en el sentido de "disposición adquirida" (Lipietz, 1983, p. 22). Este habitus fue un complemento poderoso de la planificación del Estado: él "sabía" que el sector privado seguiría muy probablemente los objetivos elegidos; recíprocamente, las empresas "sabían" que si actuaban en la dirección señalada por el Estado, crecerían y serían cada vez más poderosas -y entonces, con el tiempo, socias-. De esta manera, el Estado jugaba un rol determinante en la regulación coreana. ${ }^{7}$

En este contexto, Sakong prefiere el concepto de "reciprocidad incompleta". ${ }^{8}$ Gracias al control del crédito, las relaciones Estado-capital pueden ser definidas como una "reciprocidad incompleta" porque puede darse "la eliminación de un privilegio existente o el castigo a causa de una desobediencia" (Sakong, 1993, p. 37). No se trata de un intercambio de la misma naturaleza. La aceptación de las directivas del Estado implicaba, en contrapartida, las ayudas y los subsidios públicos; sin embargo, hace falta insistir en la existencia del segundo aspecto, "la penalización" en caso de no aceptación, que podía tener las formas más variadas: el retiro de los créditos subsidiados o incluso de los servicios públicos indispensables para la sobrevivencia de una empresa, las auditorías fiscales, el rechazo a la

6. Ver también Choi (1983, p. 331).

7. Regulación en el sentido de la teoría de la regulación francesa (Boyer, 1986) y no en el sentido anglosajón de reglamentación.

8. Se trata en este caso de nuestra traducción de la idea de "partial mutuality" de Sakong (1993, p.37).

\section{2}


participación de una empresa castigada en los proyectos estratégicos, etcétera.

Además del origen del compromiso histórico, el control estatal de los bancos explicaba también la posición subordinada del sector privado (Hart Landsberg, 1993, p. 57). A través del control estatal del sistema bancario, los "objetivos definidos por el Estado fueron cubiertos por las empresas privadas", concluye Lanzarotti (1992, p. 225). De tal manera que el rol de estas podía limitarse a ser un simple "delegado" del poder del Estado o el "principal agente" del desarrollo dirigido por el Estado (Choi, 1983, p. 329).

Los ejemplos no faltan a propósito de este rol disciplinario o de esta "reciprocidad incompleta". Song (1990) fue preciso:

El gobierno utilizó el rechazo de tales servicios [de infraestructura] para castigar a las empresas que no obedecían las políticas económicas (p. 144).

Algunos casos son ya clásicos. Uno entre ellos fue el de Hyundai. Yoo Hak-Sang reporta el diálogo entre el presidente Park y el presidente del grupo Hyundai, en 1972, cuando este pensaba abandonar el proyecto de construcción naval solicitado por el gobierno:

Si usted no puede estar en la situación de acabar una tarea así de fácil, los créditos y también los otros privilegios de los que ustedes se benefician serán inmediatamente suspendidos (citado en Rennard, 1993, p. 86).

\section{La reciprocidad incompleta y la reducción} de las incertidumbres

Por supuesto, la relación Estado-capital no se reducía a la amenaza. También a través de los privilegios o de los 
estímulos económicos y no económicos, ${ }^{9}$ “el Estado reforzaba las empresas al reducir los riesgos y las incertidumbres" (Sakong, 1993, p. 46). Aquí tenemos uno de los roles fundamentales de la intervención del Estado en la regulación coreana, durante la era Park: asegurar lo más posible la realización de las mercancías.

Encontramos aquí una especie de validación social de la producción a través del poder del Estado. Se trataba de una economía movilizada. ${ }^{10}$ La alta probabilidad de realización de las mercancías estaba integrada ex ante en el comportamiento de los empresarios elegidos o apoyados por el Estado. La incertidumbre de los "saltos peligrosos" de los valores en proceso -hacia su transformación en moneda- estaba así, en ocasiones, prácticamente eliminada, especialmente en el mercado interno; en el caso de las empresas exportadoras, el Estado colaboraba a reducir lo más posible las incertidumbres de estos saltos peligrosos, a través del apoyo a la competitividad ${ }^{11} \mathrm{o}$ a la comercialización de los bienes y servicios. En las crisis de rentabilidad de las empresas, el Estado intervenía para ayudarlas con planes de salvamento.

Podemos mostrar los casos siguientes de los astilleros y de las empresas ligadas a la producción de armamento.

En lo referente al primer ejemplo, el chaebol Hyundai estuvo a punto de rehusar definitivamente su participación en la construcción de barcos; sin embargo, después del apoyo-amenaza del Estado se decidió en 1973 a invertir en este sector. Industrias Pesadas de Hyundai (IPH), subsidiaria de este chaebol, había ya terminado, en febrero de 1974, su primer gran navío de transporte; no obstante, la

9. Por ejemplo, el prestigio de alcanzar un récord ante una meta planteada: digamos, superar el techo de exportaciones fijado. El prestigio es importante para la ética confuciana.

10. Sapir (1989, p. 47) expresa:"la movilización no suprime el test de la validación; la transfiere al organismo central".

II. A través, por ejemplo, de la política de reducción de los costos financieros o del control del costo salarial.

\section{4}


empresa tuvo problemas serios en los tres primeros años de su existencia: debido a los retardos en los plazos de entrega de los navíos solicitados, las empresas compradoras -una griega, una japonesa y otra de Hong Kong- se rehusaron a recibir cinco de ellos. Los problemas tecnológicos eran inmensos para una empresa que aprendía a producir barcos de gran calado.

La crisis era sumamente grave. Hyundai se decidió, entonces, a fundar la Compañía de Marina Mercante Hyundai para absorber los barcos no entregados y para utilizarlos en su nueva compañía de comercialización. Después el gobierno, propietario de las refinerías de petróleo del país, ordenó que toda la entrega de petróleo crudo hacia Corea fuera transportada en barcos coreanos, curiosamente en los de la Compañía de Marina Mercante de Hyundai. Superada esta incertidumbre inicial, la IPH pudo así profundizar su proceso de aprendizaje industrial y confrontar la competencia internacional con el apoyo público. Ya en 1994, jIPH era uno de los constructores de barcos más grande del mundo!

El segundo caso es el de las industrias militares. El Estado optó por utilizar el sector privado como el agente de la industrialización de la defensa, más que la industria pública. La primera fase de la substitución de las importaciones militares inició en 1969, bajo Park. La incertidumbre de las empresas privadas fue reducida al mínimo. El Estado garantizaba la sobrevivencia de los contratistas privados de productos militares a través de pedidos de largo plazo, con la promesa de salvamento de los contratistas que cayeran en números rojos y, en ocasiones, ¡hasta con el pago por adelantado hasta de $90 \%$ de la factura! (Moon, 1991, p. 449). ${ }^{12}$

12. En 1988, había ochenta y dos empresas aceptadas por el gobierno como productoras de artículos de defensa y alrededor de mil quinientas subcontratistas. Ese mismo año, ocho de los diez chaebols más grandes, tenían ya intereses en esta industria. 


\section{El poder creciente de los chaebols ${ }^{13}$}

Esta política practicada por el Estado generó las condiciones mismas de imposibilidad de mantener el aspecto autoritario de su relación con las empresas. Las autoridades eligieron a los chaebols como los actores principales de la industrialización coreana; veían ventajas de competitividad al mantener y apoyar a los grandes conglomerados. De esta manera, gracias al apoyo público, el poder económico de los chaebols se reforzó cada vez más. En el análisis de la misión del Banco Mundial enviada a Corea en 1985, la participación de los treinta chaebols más grandes en las ventas de productos manufactureros, se multiplicó en los años ochenta y ya en 1978 representaba $34.1 \%$ del total. ${ }^{14}$ Las ventas de los diez chaebols más grandes representaban, en 1974, 15.1\% del total; y, en 1978, 30.1\% del PNB. ${ }^{15}$

Desde principios de los años setenta, encontramos signos del poder de negociación de los chaebols. En 1971 y 1972, bajo el acuerdo de estabilización firmado con el FMI, el acceso al crédito exterior fue limitado seriamente y el crédito de las instituciones bancarias de hecho fue racionado. La situación financiera de los chaebols fue sometida así a un choque considerable. Su opción era buscar el crédito en el mercado paralelo, con tasas de interés muy elevadas. La Federación de Industrias Coreanas, representante de los chaebols, demandó al gobierno, en 1971, el cambio de esa política y una moratoria de sus deudas contratadas en el

13. Estos conjuntos empresariales tienen tres características fundamentales: a) son conglomerados; b) poseen empresas en diversas ramas, de tal manera que su actividad es muy diversificada; c) son dirigidos por los miembros de la familia del fundador (ver Steers, Shin y Ungson, 1989, pp. 33-48).

14. La estructura del mercado coreano de manufacturas, ya muy concentrado antes de 1970, se concentra aún más entre 1970 y 1977; por ejemplo, los productos controlados en más de $80 \%$ de las ventas por una sola empresa pasaron de representar $29.6 \%$ del total de los productos a $31.6 \%$ en 1977 (ver WB, 1987, pp. 28-32).

15. Ver Kim, Seok-Ki citado en Amsden (1989, p. I I6).

\section{6}


mercado paralelo. El 3 de agosto de 1972, Park las aceptó y decretó una moratoria de tres años a favor de las empresas deudoras. ${ }^{16}$

Podemos observar la participación de los diez chaebols más grandes en el valor añadido total: de 1973 a 1978, durante el periodo del boom de las industrias pesadas y químicas, su aporte se duplicó y pasó de 5.1\% a 10.9\% del PIB.

Aunque había una "disposición adquirida" en cuanto a la intervención del Estado y a las relaciones Estado-capital, de ahora en adelante sería difícil para el primero perseverar en una política especialmente penalizadora o autoritaria frente a los chaebols.

En síntesis, encontramos una relación singular Estadocapital marcada por el compromiso histórico de 1961, concluido bajo la amenaza. El modo de regulación coreano incluía así una fuerte dosis de coerción estatal pero también de incentivos y de reducción de riesgos. En primer lugar, el Estado disciplinó a los empresarios y los constriñó a seguir la planificación; después, el Estado respaldó sobre todo a los chaebols y los reforzó al reducir las incertidumbres. Se trataba pues de una reciprocidad incompleta. Sin embargo, esta relación provocó el crecimiento del poder de los grandes conglomerados. El control de los chaebols se volvió desde entonces más difícil, aunque el Estado seguirá manteniendo el arma financiera. Los conglomerados estaban adquiriendo mayor poder económico, se estaban convirtiendo en gigantes, pero aún con pies de barro... financieros.

\section{El crecimiento del poder de los chaebols en el periodo del ajuste 1979-1986}

Respecto de este periodo, diversos analistas, tanto economistas como politólogos, comienzan a hablar de cambios en 
la relación Estado-empresarios. La relación de "reciprocidad incompleta" estaría así en un proceso de modificación, dado que el poder de los chaebols era cada vez más perceptible. Woo (1991, p. 175) se distingue incluso en lo que se refiere a la separación típica sector privado-sector público en Corea, en un marco de incremento del poder de los chaebols:

[...] suponemos que los sectores público y privado han actuado como si fueran uno, en lo que podemos llamar 'Korea, Inc.' (p. 175).

En realidad, las estadísticas permiten concluir que el poder económico de los chaebols se incrementó durante este periodo de ajuste. Incluso la conclusión de Amsden (1989, pp. 116, 136) es que este periodo de liberalizaciones "contribuyó al aumento y no a la disminución de la concentración económica”. Las ventas de los diez conglomerados más grandes, que representaban $32.8 \%$ del PNB, ¡llegaron a significar $67.4 \%$ en 1984 ! El valor añadido de estos pasó de $10.5 \%$ del PIB en 1979 ¡hasta 24.1\% en 1985! De esta manera, la salud de la economía coreana se mezcla con la solidez de los chaebols.

El Estado contribuyó a esta concentración a través de la política de las fusiones forzadas en los programas de reestructuración industrial. Las grandes empresas fueron obligadas a fusionarse a cambio de favores fiscales (Sakong, 1993, pp. 76-77). Los conglomerados fueron los compradores principales de las empresas en dificultad.

Además, aunque el Estado haya dirigido la privatización bancaria para evitar el control de los bancos nacionales por los chaebols,${ }^{17}$ estos aprovecharon la liberalización de las instituciones financieras no bancarias: por ejemplo, de las veintiséis compañías de seguros, once eran propiedad 
de algún conglomerado a fines de 1983 (Bello y Rosenfeld, 1990, p. 70). ${ }^{18}$ De acuerdo a Amsden (1989, p. 134) y a Bello y Rosenfeld (1990, p. 69), el Estado concedió a los chaebols, a cambio de su aceptación de la apertura comercial, una muy amplia posibilidad de control de las instituciones financieras. Los chaebols tendrán así también una posibilidad de dar la vuelta a los controles estatales del crédito.

Sin embargo, el Estado pudo aún imponer soluciones en el caso de las reestructuraciones industriales. Además, a pesar del poder creciente, industrial y financiero de los conglomerados, los ejemplos de penalización por la noobediencia no desaparecieron. Testimonia esta situación el caso del grupo Kukje.

En 1984, este grupo -que era nada menos que el séptimo chaebol-se rehusó a colaborar más generosamente con el Movimiento de la Nueva Comunidad ${ }^{19}$ y a participar en una campaña de financiamiento de la Fundación Ilhae. ${ }^{20}$ ¿Cuál fue el resultado? En diciembre de 1984, el Korea First Bank, privatizado en 1982, se rehusó a pagar los cheques de la empresa Kukje, rechazo que generó una bola de nieve y acorraló al grupo a la bancarrota (Steers, Shin y Ungson, 1989, pp. 45-46 y Bello, 1990, p. 52).

Un funcionario del Banco de Corea nos señaló que la deuda del grupo Kukje había sido refinanciada varias veces por los bancos comerciales y en una ocasión por el banco central mismo (entrevista personal, 1992). El presidente

18. Las instituciones financieras no bancarias fueron cada vez más importantes en la captación de ahorro -más que en manejo de los créditos-: en 1976 captaban ya $32.8 \%$ del ahorro, en $198551 \%$ (BOK, 1990). El poder de los chaebols en estas instituciones iba de más en más fuerte. En 199I, los treinta conglomerados más grandes controlaban ya $45 \%$ de estos intermediarios financieros no bancarios (Sakong, 1993, p. 63).

19. Este movimiento fue creado por el gobierno como una organización de ayuda para el desarrollo rural y era dirigido en 1984 por un hermano del presidente Chun Doo Hwan.

20. Organización para la búsqueda de la reunificación coreana y próxima al presidente Chun (Shin, 1989). 
de Kukje no atendió los consejos de los funcionarios de la banca central en el sentido de reestructurar su deuda (los créditos de corto plazo tenían un peso muy importante); al contrario, la empresa se endeudó todavía más con créditos a corto plazo para un proyecto de construcción. Si Kukje se hubiera endeudado a largo plazo no habría tenido problemas, pero "no respetó los consejos del gobierno" (entrevista personal, 1992), concluyó nuestro entrevistado. De acuerdo a otro banquero, si el gobierno se decidía por cerrar una compañía, podía hacerlo fácilmente y "todo lo que debía hacer era: llamar a los bancos comerciales coreanos, que él controla, y exigirles no refinanciar la deuda de la compañía" (citado en Bello, 1990, p. 52).

Por otra parte, los chaebols comenzaron a protestar públicamente contra las órdenes del Estado. En marzo de 1985, un grupo de los industriales más ricos de Corea se reunió con los funcionarios públicos más importantes y presentó un verdadero listado de demandas: el gobierno no otorga financiamiento suficiente ${ }^{21} \mathrm{y}$ al mismo tiempo exige la reestructuración de los conglomerados; el gobierno mantiene un sistema financiero mediocre, se rehúsa a verdaderamente privatizar los bancos comerciales nacionales y crea la inestabilidad a través de estándares arbitrarios y decretos bizarros; en abril de 1982, Chong Chu-Yong, líder de Hyundai y uno de los industriales más influyentes del país, reclamó públicamente el fin del intervencionismo del Estado y la autonomía real del sector financiero (Woo, 1991, p. 200). Fue el inicio de la rebelión de los privilegiados del Estado.

De acuerdo a Hart-Landsberg (1993, p. 230-237), en estos años pudo discernirse un debilitamiento del poder del Estado: los chaebols eran más independientes y lograron escapar a algunas de las directivas del Estado. Por ejemplo, 
Estado y empresarios en Corea del Sur: entre la reciprocidad y la autonomía

tres conglomerados se opusieron a participar en las fusiones forzadas de las reestructuraciones industriales. Los chaebols comenzaron así a cuestionar el elemento incompleto de la reciprocidad.

¿Hacia un nuevo compromiso? Una década de fuertes cambios: 1987-1997

La rebelión democrática de 1987 generó también fuertes críticas contra la concentración del poder económico, lo que afectó la relación Estado-conglomerados. A fines de los años ochenta, el valor agregado de los diez chaebols más importantes se situaba en alrededor de una cuarta parte del PIB, aunque había comenzado a disminuir ligeramente a partir de 1986 (Sakong, 1993, p. 247).

Ante las nuevas realidades impuestas por el proceso de democratización, especialmente las fuertes críticas a la concentración de poder económico y el activismo sindical (Valencia, 1996), los empresarios coreanos se dotaron en 1989 de una organización cúpula, la Federación Nacional de Organizaciones Empresariales. ${ }^{22}$

Un ejemplo significativo en cuanto a la opinión pública coreana es el malestar de diversos sectores a causa de la desviación especulativa de recursos financieros de los chaebols hacia la compra de terrenos: ${ }^{23}$ en $1989,65 \%$ de

22. Formada por seis grandes organizaciones empresariales, incluida la representante de los conglomerados, Federation of Korean Industries: Korean Chamber of Comerce and Industry, Korean Foreign Trade Association, Korean Federation os Small Businesses, Korean Employers Association y Federation of Korean Banks (Eckert, 1993, p. II2).

23. Cuando le preguntamos a un representante sindical su punto de vista en cuanto a problemas de competitividad, nos respondió (entrevista personal, 1993B): "Estamos de acuerdo en cuanto a que hay una crisis en la economía coreana. Cuando se habla de productividad, hay que distinguir productividad del trabajo y del capital [...] Una tercera parte del capital es invertida en el mercado negro, en la especulación. El capital no es sano, no es invertido en la productividad, sino en la especulación: casas, terrenos”. 
las tierras en propiedad privada pertenecían solamente a 3\% de la PEA, en particular a los conglomerados; en 1989, entre los propietarios, solamente $10 \%$ poseían $76.9 \%$ de los terrenos. Así, la apropiación de tierras estaba mucho más concentrada que el ingreso: el índice de Gini para la primera era 0.85 y para el segundo 0.36\% (Sakong, 1993).

En este contexto, el Estado multiplicó en el periodo posterior a 1987 diversos proyectos para tratar de controlar a los chaebols. En 1991, el régimen de Roh trató de obligar a los conglomerados hacia la especialización. En 1990, el presidente Roh Tae Woo había ordenado a los cuarenta y nueve conglomerados más importantes vender una parte significativa de sus propiedades inmobiliarias y amenazó a los cinco primeros con investigaciones de carácter fiscal si compraban propiedades para especular; sin embargo, los resultados fueron débiles: el Estado no pudo imponer una solución a los chaebols (Hart-Landsberg, 1993, pp. 239-240). Incluso el Plan para una Nueva Economía (1993-1997) habla de nuevo de modificar la estructura fiscal para imponer nuevos impuestos a los propietarios de más de una casa (Chang, 1993, p. 6) y a los propietarios de terrenos.

La respuesta de algunos conglomerados no tardó, especialmente la del líder de Hyundai, Chong Chu Yong. En 1992, Chong se lanzó en la batalla electoral por la presidencia, frente al candidato oficial -Kim Young Sam-y el de oposición -Kim Dae Jung-. Durante su campaña, hizo públicas las cantidades de dinero que había dado al partido oficial y se decidió por desafiar al candidato oficial y antiguo opositor, Kim Young Sam (Fields, 1995, pp. 60-61). Es el símbolo de una relación Estado-empresarios en transición. Pero, ¿hacia dónde?

Il Sakong escribió en esos años: 
Estado y empresarios en Corea del Sur: entre la reciprocidad y la autonomía

El asunto del crecimiento de los chaebols -a saber, la concentración del poder económico- es todavía uno de los temas más espinosos de Corea en la actualidad (Sakong, 1993, p. 63).

En ese periodo la situación no era clara. Las posiciones del gobierno no eran uniformes. Entrevistamos en 1993 a un funcionario del Ministerio de Planificación, que trabajaba sobre el asunto chaebol, y a un miembro del Comité Monetario. El primero nos había afirmado que a la larga el Estado disolvería los conglomerados, como Japón lo hizo con los zaibatsu. Al contrario, el segundo nos señaló lo siguiente:

En cuanto a los chaebols no hay que exagerar. Ellos tienen lados positivos para la economía coreana: al nivel de la tecnología, de la competitividad, de las exportaciones y del empleo. Pero ellos tienen también su lado negativo: el control del mercado. Sin embargo, en el contexto actual de la crisis económica, es preciso cuidarse de atacarlos (entrevista personal, 1993A).

Las políticas de ajuste de los años ochenta convergieron en profundizar las transformaciones iniciadas en los años setenta. La parte del valor agregado de los conglomerados en el PIB permaneció aún enorme y estos se reforzaron con las fusiones forzadas de empresas en dificultad financiera y, además, los treinta chaebols más grandes controlaban, en 1991, 45\% de los intermediarios financieros no bancarios. Arrancando de un estatus subordinado, los conglomerados exigen cada vez más un estatus de socio en la dirección de la economía nacional. Muchos investigadores hablan de Korea, Inc. o de la joint-venture Estado-chaebols.

De acuerdo a Shim (1992, p. 216), de 1962 a 1981 el Estado elegía los grandes proyectos y los programas específicos, pero después de las políticas de ajuste y más, precisamente desde 1982, el Estado indica solamente las grandes líneas de los programas y delega el resto a las 
empresas. ${ }^{24}$ Peter Evans (1995) destaca esta relación para los años ochenta en el sector de la información y las telecomunicaciones -seleccionado desde la época de Park Chung Hee como uno de los sectores estratégicos a desarrollar-. ${ }^{25}$

Varios investigadores coinciden con la periodización de Shim. También la versión oficial fue la misma en la revisión del V Plan Quinquenal en 1983. Para Yoo (1991, p. 284), del Instituto Coreano del Desarrollo (KDI), incluso desde 1979 hubo una disminución de la intervención gubernamental. Esta visión coincide con el análisis del miembro del Comité Monetario que entrevistamos en abril de 1993: a la pregunta de en cuáles ramas industriales podrían especializarse los exportadores coreanos, nos respondió: "tocará a las empresas elegir" (entrevista personal, 1993A). Así, según Amsden (1989, p. 88), el rol de empresario ha devenido menos un monopolio del Estado y más una joint-venture Estado-conglomerados; en la relación Estado-empresarios sobresaldrá el rol de cuidador (husbandry) de Evans (2005).

En conclusión, si es cierto que hubo una disminución de la intervención del Estado en la economía coreana, este mantenía aún un rol determinante en la regulación coreana: el poder de las autoridades en esta época, tanto en la asignación del crédito como en la planeación económica, era todavía determinante. Las políticas de ajuste no destruyeron estos aspectos institucionales, aunque el Estado debe negociar cada vez más con los chaebols. No obstante, la democratización abrió también flancos de debilidad a los conglomerados.

24. No es la conclusión de Steers, Shin y Ungson (1989, p. 19). De acuerdo a ellos, las autoridades eligen aún las ramas prioritarias y también las empresas.

25. Evans lo muestra con el estudio del 4-Megabit DRAM Project en el área de semiconductores y del Electronic switching systems en el área telefónica. Con el apoyo del Ministerio de Comunicaciones para el desarrollo tecnológico (investigación y créditos), con la protección de los mercados y el posterior impulso a la exportación, el Estado asociado a los conglomerados impulsó notablemente el desarrollo del sector de telecomunicaciones (ver los detalles en Evans, 2005). 
¿Cuál fue el desarrollo de esta relación a partir del gobierno de Kim Young Sam? ¿Siguió los viejos patrones jerarquizados de un Estado dirigente?

En el largo plazo, las autoridades coreanas prefirieron un enfoque gradual en diversos procesos de liberalización, en especial el comercial y el financiero (Valencia, 1996). Sin embargo, con la propuesta de Se Gye Hwa (globalización) de Kim Young Sam se dieron cambios bruscos especialmente en la liberalización financiera y en la forma de intervención del Estado a través de la política industrial (Shin y Chang, 2003; Chang, 2004), que permitieron un mayor fortalecimiento de los conglomerados (Shin, 2004, p. 140) a pesar de que se mantuvieron algunas disposiciones para limitarlos.

Kim Young Sam trató, entre otras acciones, de limitar el acceso preferencial de los conglomerados al crédito bancario interno y buscó la especialización de los mismos para evitar la diversificación de sus actividades (Shin, 2004). Sin embargo, los procesos de liberalización posibilitaron el robustecimiento en el corto plazo de los conglomerados frente a las autoridades. El gobierno de Kim permitió mayores entradas al mercado financiero en el sector no bancario y liberalizó la cuenta de capitales -en especial, la entrada de capitales de corto plazo- en el marco del ingreso de Corea a la OCDE (OECD, 1993). Estas medidas disminuyeron fuertemente la capacidad del Estado para influir en los conglomerados a través del manejo de los instrumentos financieros (crédito); los chaebols pudieron recurrir a endeudamientos masivos en el exterior, con préstamos de corto plazo, y a recursos internos mayores. Por otra parte, Kim Young Sam también relajó notablemente la política industrial al renunciar a las iniciativas de coordinación de las inversiones. ${ }^{26} \mathrm{Y}$ los chaebols siguieron presionando para

26. En 1993 permitió a Samsung la entrada a la ya saturada industria automotriz (Shin y Chang, 2003; Chang, 2004). 
debilitar la capacidad estatal de limitarlos o controlarlos: por ejemplo, en 1997 la FKI publicó un informe ultraliberal en el que demandaba un extremo retiro del Estado de la economía e incluso el cierre de la mayor parte de los ministerios (Shin y Chang, 2003).

Así, en el periodo inmediatamente previo a la crisis, los conglomerados equilibraron más sus relaciones con el Estado, al existir más posibilidades de encontrar fuentes de financiamiento tanto en el mercado interno -a través de las instituciones no bancarias- como en el externo -endeudamiento de corto plazo- y de definir proyectos de inversión también en el mercado interno como en el externo -fuerte aumento de las inversiones en el extranjero-. No obstante, la que podría considerarse como una época de fortalecimiento de los chaebols -mediados de los noventageneró inmediatamente una severa contradicción: los conglomerados se debilitaron estructuralmente debido a un sobreendeudamiento, ${ }^{27}$ a la crisis monetaria -devaluación del won- y a los problemas de las empresas subsidiarias en Asia del Este en 1997. En 1997 varios chaebols vivieron episodios de insolvencia y quebraron. ${ }^{28}$ Además, se debilitaron políticamente debido a las fuertes críticas del FMI y de importantes sectores de la sociedad coreana, cuestionamientos que se sintetizaron en la presunción del crony capitalism (Kang, 2002). Los fuertes cuestionamientos a los grandes empresarios se agudizaron durante la crisis de 1997, pero tienen fuertes raíces históricas de acuerdo al

27. Los ratios de endeudamiento de los treinta conglomerados más importantes pasaron de 347.5 en 1995 a 519 en 1997. Incluso en cuatro casos superaba el ratio de I 000 (OECD, I999, p. I38). Shin y Chang (2003), con elementos comparativos, relativizan esta idea del sobreendeudamiento que necesariamente llevaría a una crisis de la economía coreana y de los mismos chaebols.

28. Seo (2000, p. 5I8) proporciona una lista de nueve, entre los que destacan la acerera Hanbo -conglomerado número catorce- y la automotriz Kia -número diecinueve-.Ver también a Shin y Chang (2003). 
Estado y empresarios en Corea del Sur: entre la reciprocidad y la autonomía

profesor Eckert (1993): los empresarios no han logrado un liderazgo moral y filosófico en la sociedad coreana.

¿Crisis financiera y gobiernos "progresistas", crisis del viejo compromiso? (1997-2008)

La relación Estado-conglomerados, se ha ido convirtiendo en un asunto muy sensible en la vida económica y política de Corea del Sur; de hecho, el asunto chaebol y el conflicto distributivo son, en algunos sentidos, parte del mismo problema. En un estudio realizado en los años de la crisis, de acuerdo a una gran mayoría de los entrevistados el factor que más contribuyó a esta situación fue la cercana relación entre gobierno y conglomerados -la "amigable relación entre gobierno y empresas" en 78\% de las respuestas (Hayo y Shin, 2002)-. ${ }^{29}$ Paradójicamente, la extraordinaria fuerza económica de los conglomerados generó las bases de su propio debilitamiento político.

Asunto tan central que para algunos investigadores en ella se encuentra parte de la explicación de la crisis financiera de 1997: para Meredith Woo-Cumings (1999, p. 3), por ejemplo, el origen de la crisis financiera reciente habría estado en la falta de habilidad para resolver el nudo gordiano entre el Estado y el sector bancario por una parte, y el sector corporativo enormemente endeudado por la otra; y para You y Lee (1999), la equivocada secuencia de apertura financiera -primero los capitales de corto plazo, luego los de largo plazo-, origen de la crisis, sería una medida en beneficio de los grandes conglomerados y en perjuicio de la economía en su conjunto. Asunto tan sensible que la concentración de poder económico en unos cuantos conglomerados ha ido generando una polémica entre los democratizadores - tal concentración sería una amenaza a

29. Ver Valencia, 2012. 
la democracia (Kwark, 2000)-, ${ }^{30}$ los reformadores de libre mercado internos y externos (la corporate governance de esta "reciprocidad incompleta" estaría llena de beneficios no transparentes y de apoyos fuera del mercado) y entre algunos de los defensores de la política industrial coreana (los conglomerados serían una fortaleza competitiva y tecnológica de la economía coreana). ${ }^{31}$

Diversos investigadores, desde horizontes teóricos a veces contradictorios, señalaron la acumulación de exceso de capacidad en algunas industrias en el periodo inmediatamente anterior a la crisis, que generó problemas de rentabilidad en los grandes grupos industriales y debilidad financiera (Chang, 2003; You y Lee, 1999). Economistas del FMI encontraron también ambiciosos proyectos emprendidos por los chaebols entre 1993 y 1996 (Chopra, 2002). Economistas del mismo Banco Central cuestionaron los fuertes incentivos para expandir las inversiones, sin cuidado ni atención a los riesgos, otorgados por el alto nivel de protección dado por el gobierno a los conglomerados a cambio de que estos jugarán el rol de locomotora de la economía (BOK, 1998).

El FMI mismo empujó a la reforma chaebol, con la exigencia directa de que disminuyeran radicalmente su tasa de endeudamiento, origen para él de la debilidad financiera de Corea del Sur, y una mayor liberalización de la cuenta de capitales. ${ }^{32}$ El mismo Kim Dae-Jung consideró, por un lado, que la represión financiera había sido una manera de controlar al sector empresarial (Woo-Cumnigs, 1999) y por otro, que el disfuncionamiento de la economía y del sector

30. La transparencia y la lucha contra la corrupción son demandas de los movimientos ciudadanos y de las ONG; entre ellas se encuentra el cambio del "sistema regulatorio estatal hacia un sistema de coordinación industrial y financiera que corte con la colusión entre políticos y empresarios” (Yi, 1998, p. I30).

3I. Ver en particular a Chang, 2004.

32. Un equipo de economistas del FMI, trabajando en Corea, señaló que la debilidad estructural del sector financiero y de los corporativos en el marco de la crisis de 1997 estaba interrelacionada (Chopra, 2002).

\section{8}


financiero tenía un déficit democrático debido a la colusión entre hombres de Estado y empresarios (Kwark, 2000). ${ }^{33}$ Por las dos razones, este presidente favoreció una mayor liberalización financiera. En entrevista con un académico cercano a Kim Dae-Jung, a mediados de 1999, le preguntábamos la razón por la que este adoptaba un lenguaje muy cercano al FMI en cuanto a las reformas económicas y a las liberalizaciones necesarias. ¿Se trata de real confluencia ideológica con el FMI o de un "velo" discursivo como señalara Wade (1999, p. 21)? Según nuestro entrevistado, se debió a que el presidente estaba decidido a disminuir el poder de los chaebols y a que, en las condiciones postcrisis, encontraba en el FMI a un aliado (entrevista personal, 1999).

Tras el viejo compromiso Estado-empresarios (chaebols) estaba una fuerte coalición pro crecimiento económico a toda costa en la que estaban inicialmente los gobiernos militares (y después el primer gobierno civil tras la apertura democrática), la burocracia fortalecida en los años de crecimiento, los grandes conglomerados y como socio menor los sindicatos controlados a través de la Federación Coreana de Trabajadores (FKTU, oficialista), además de los sectores medios (Choi, 1983), beneficiarios del estado desarrollista y permeables ante las posiciones conservadoras y la narrativa anticomunista. Esta coalición, ya en crisis desde 1987, había ya llegado a sus límites (López Aymes, 2005, p. 198) y el gobierno de Kim Dae-Jung tenía otros aliados: una creciente coalición prodistribución que cuestionaba la acumulación de poder y riquezas en los grandes conglomerados; a esta coalición se fueron integrando, entre otros sectores, diversas organizaciones civiles ${ }^{34}$ y la organización autónoma

33. Woo-Cummings (1999, p. 27) habla de "raketeering state". En la relación Estado-chaebols se fue favoreciendo o generando un sistema de presiones para aportaciones políticas (cuasi-mpuestos).

34. Por ejemplo, la organización conocida como People's Solidarity for Participatory Democracy (PSPD) fue muy activa en un movimiento de defensa de los accionistas minoritarios de las grandes empresas; en 1998 ganó una demanda 
de sindicatos (Confederación Coreana de Trabajadores, KCTU) (Valencia, 2012). La crisis económica de 1997 fue al mismo tiempo crisis de legitimidad de los chaebols y de su capacidad de liderazgo moral de la sociedad como diría Eckert (1993): fue tal que para algunos cercanos al círculo de Kim Dae Jung la visión era que varios empresarios habían acumulado su riqueza de manera ilegal, como "criminales" (entrevista personal, 2008A).

Así, el gobierno liberalizó la entrada de capitales: la inversión extranjera directa (IED) aumentó notablemente; ${ }^{35}$ y emprendió una reestructuración del sector corporativo en el marco de las políticas de ajuste acordadas con el FMI. De nuevo, tradición se impone, el gobierno de Corea tomó un activo papel en esta reestructuración (Stiglitz, 2002, p. 127). Economistas del FMI y del BM aceptaban la necesidad de esta participación del Estado en la reestructuración, pero concluyeron que aún deberían cerrarse empresas no viables de los conglomerados, que las autoridades deberían evitar acciones que sostuvieran la vieja idea de que los chaebols son "too big to fail" (TBTF) ${ }^{36}$ y cuestionaron que el organismo encargado de la supervisión financiera (FSC, siglas en inglés de Financial Supervisory Commission) hubiera incitado a los bancos ya sea a eliminar de la lista de acreditables a cincuenta y cinco compañías o a otorgar créditos a empresas en problemas (Chopra, 2002, pp. 68 y 83; Mako, 2002, pp. 210-225); de nuevo aparecieron las amenazas de corte de

\footnotetext{
en contra del Korea Jirst Bank (Kalinowski, 2009, p. 295). Con respecto al peso del PSDP en la coalición pro distribución puede verse Valencia (2012).

35. Hasta 2002 se habían acumulado 49200 millones de dólares de IED; sólo en el periodo 1997-2002 entraron 32400 millones, prácticamente dos terceras partes de lo acumulado hasta 2002 (Kim, 2003, p. 2008)

36. La tesis de TBTF debe cuestionarse en el caso de Corea de acuerdo a Shin y Chang (2003, pp. 47-49), porque históricamente se dieron casos de quiebras de chaebols: tres de los principales diez en los años sesenta (Samho, Gaepoong y Donglip), tres de los principales treinta entre 1990 y 1996 (Hanyang, Yoowon y Woosung) y en plena crisis de 1997, seis de los principales treinta (Kia, Halla, Jinro, Hanbo, Sammi y Haitai).
} 
crédito a las empresas reticentes (Wade, 1999, p. 21; OECD, 1999; Leem, 2000) y apoyos a empresas en problemas.

Durante el periodo de Kim Dae Jung, el Estado buscó equilibrar las relaciones con los grandes conglomerados. Aprovechó la oportunidad de la crisis para contenerlos, pero no rompió la comunicación con ellos. ${ }^{37} \mathrm{El}$ gobierno les propuso un Big Deal para que se especializaran y para evitar las sobreinversiones; las autoridades promovieron la reestructuración financiera de los chaebols, de la que Daewoo fue el caso ejemplar. ${ }^{38}$ Debe recordarse, sin embargo, que estos planes de reestructuración de los conglomerados no son los primeros: ya ha habido varios desde los años ochenta (Valencia, 1996) y no todos han resultado exitosos (López Aymes, 2005). El balance de la reestructuración propuesta por Kim Dae Jung es mixto: por una parte, efectivamente logró una reducción notable de la tasa de endeudamiento (debt to equity ratios) de las empresas manufactureras, que se redujo sustancialmente de 396 en 1997 a 135 en 2002; este indicador llegó a 122 en el caso de los treinta mayores conglomerados (Kim, 2003, pp. 195 y 207), frente a 519 en 1997 (OECD, 1999, p. 138). Por otra parte, en algunas ramas industriales el Estado logró, a través del Big Deal con los cinco principales conglomerados, superar los problemas de sobreinversión y exceso de capacidad instalada; sin embargo en los casos estratégicos de semiconductores, petroquímica e industria electrónica, en 2002 no había concluido la reestructuración (Kim, 2003, pp. 262-264). En 2001 el gobierno introdujo un plan de salvamento financiero para los conglomerados en problemas, especialmente Hyundai, que puede entenderse como que el "entusiasmo gubernamental por

37. Ver en LópezAymes (2005, p. 198, nota 56), diferentes rondas de conversaciones del presidente con los dirigentes de cinco grandes conglomerados.

38. Puede verse en Kim (2003, p. 254) una síntesis de las propuestas gubernamentales de reestructuración corporativa durante el gobierno de Kim Dae Jung, en especial entre 1998 y 2000. 
la reestructuración del sector corporativo se fue enfriando rápidamente" (Kim, 2003, p. 260). Se generaron, por último, diversos cambios en la gobernanza corporativa, que Shin y Chang (2003) caracterizan como acercamiento al modelo angloamericano de "business firm", con elementos positivos de transparencia y defensa de los accionistas minoritarios, pero con pérdida de la capacidad de toma de riesgo de los chaebols, que les había permitido avances tecnológicos.

En el gobierno de Roh Moo-Hyun se continuó con el enfoque de fortalecimiento del Estado frente a los conglomerados y con la política redistributiva (incremento de los gastos sociales). ${ }^{39}$ En el antiguo esquema del Estado desarrollista, las autoridades públicas buscaban -o imponían-acuerdos generales con los conglomerados para invertir en diversos sectores, para especialización de los conglomerados en particular y para evitar sobreinversión y sobreproducción (Shin y Chang, 2003); en el esquema modificado después de los años noventa y las reestructuraciones de fines de esa década e inicio de la siguiente, se buscaron formas de asociación en sectores estratégicos con múltiples empresas (no sólo los chaebols), que permitieran desarrollar proyectos de alta tecnología; ${ }^{40}$ el caso estratégico de telecomunicaciones ${ }^{41}$ es ejemplar al respecto con el proyecto denominado

39. El gobierno de Roh también emprendió la negociación de un acuerdo de libre comercio con Estados Unidos, lo que le generó un serio distanciamiento y ruptura con partes de la coalición distributiva mencionada anteriormente: organizaciones civiles y académicos progresistas militaron en contra de este tratado y cuestionaron fuertemente al presidente Roh.

40. Ver la comparación con el caso mexicano en relación a telecomunicaciones en Salas Porras (2007). A pesar del gran crecimiento de TELMEX -y de la riqueza de Carlos Slim-, el sector de telecomunicaciones sigue dependiendo de la importación de teléfonos y de tecnología.

4I. Puede verse en $\operatorname{Lim}(2010$, p. 198) el peso del sector de la información y las telecomunicaciones en 2007 en el PIB (33.5\% del PIB, por lo que se refiere a la industria y a sus aplicaciones), en las exportaciones (33.7\% del total) y en penetración en el mercado interno ( $89.7 \%$ de la población con celular y $80.6 \%$ de los hogares con internet de banda ancha, el primer lugar en el mundo al respecto).

\section{2}


IT839, impulsado por el Ministerio de la Información y la Comunicación (MIC) ${ }^{42}$ a partir de 2004 (Kim, 2012 A y B).

Para impulsar la producción y exportación de teléfonos celulares a gran escala, el MIC, junto con empresas coreanas, apoyó el desarrollo de servicios de segunda generación basados en la tecnología de comunicación inalámbrica conocida como Code Division Multiple Acces (CDMA), generada por la empresa estadunidense Qualcomm (Kim, 2012 B). ${ }^{43}$ Esta empresa aceptó transferir tecnología a las empresas coreanas, a cambio por supuesto de regalías por la utilización de la parte más avanzada -el sistema incorporado en un chip- de los teléfonos portátiles; empresas como Samsung y LG produjeron versiones avanzadas del CDMA en acuerdo con Qualcomm. Esta a su vez aceptó, en acuerdo firmado en 1992, regresar 20\% de las regalías obtenidas en quince años, a la institución pública ETRI (Electronic and Telecommunication Research Institute). Las empresas coreanas pudieron así dominar en el mercado interno de celulares, hasta con un $90 \%$ del mercado, y se convirtieron en líderes internacionales gracias al dinamismo de sus exportaciones (Hira, Morfopoulos y Chee, 2012); sin embargo, el superávit en el comercio exterior del sector de telecomunicaciones fue muy pequeño (menor a cincuenta millones de dólares previo al conflicto con Qualcomm en 2001). Las empresas coreanas habían logrado escalar tecnológicamente pero todavía como seguidoras y no como desarrolladoras de alta tecnología de punta. Desde la época del gobierno de Kim Dae Jung, en los

42. En 1994, el EPB fue fusionado con el Ministerio de Finanzas, con lo que se debilitó la capacidad institucional gubernamental para la planificación económica y la coordinación de inversiones; sin embargo, en ese mismo año el gobierno de Kim Young Sam decidió ampliar las funciones del Ministerio de Comunicaciones (MOC) con la adición de la responsabilidad de coordinar las acciones en torno a los sectores de información y software, para enfrentar los retos de la Se Gye Hwa para el sector clave da las telecomunicaciones. Este Ministerio ampliado fue denominado MIC (Kim, 20I2 A, pp. I52-153).

43. Seguimos largamente a Kim (2012 B) en la presentación de este caso del sector de telecomunicaciones. 
medios se hicieron públicas críticas a la empresa Qualcomm: no cumplió parte de los acuerdos de regresar un porcentaje de regalías a ETRI y en cambio las empresas coreanas le habían pagado fuertes regalías; más tarde, en 2004, se supo que Samsung había desarrollado una versión mejorada del CDMA (la versión CMDA 1x EV-DV), pero que Qualcomm se había opuesto a su comercialización. Incluso, la asociación privada Korea Industrial Technology Association dio a conocer que sólo en 2002 las empresas coreanas habían pagado por regalías 2700 millones de dólares (no sólo a Qualcomm sino también a IBM y Microsoft) (Kim, 2012 B).

En este marco, en 2004, el gobierno de Roh impulsó el Proyecto IT839, que incluía entre otros aspectos la promoción de ocho nuevos servicios de alta tecnología y nueve motores de crecimiento tecnológicos (Kim, 2012 A, p. 146). Entre los servicios incluidos estaba el WIPI (Wireless Internet Plataform for Interoperability, para generar interoperabilidad entre las compañías operadoras) y la tecnología DMB (Digital Multimedia Broadcasting, tecnología generación 3.5 de comunicación inalámbrica para transmitir emisiones televisivas de antenas terrestres a teléfonos móviles). ${ }^{44}$ En cuanto a la propuesta de WIPI, había en Corea tres operadoras de servicios de internet inalámbrico -Korea Telecom Freetel, SK Telecom y LG Telecom-, cada una con plataformas diferentes para "bajar" aplicaciones de software y sin interconexión; en 2001, Qualcomm había introducido su propia plataforma BREW (Binary Run-Time Environment for Wireless) a través de Korea Telecom Freetel (Kim, 2012 B). En cuanto a DMB, existían en el mercado coreano tecnologías de empresas extranjeras, como Qualcomm y Nokia (Kim, 2012 A).

La idea de fondo a través de WIPI y DMB era desarrollar tecnología para que las empresas coreanas dejaran de ser

44. Seguimos de nuevo a Kim (20I2 A y B), con respecto a WIPI y DMB.

\section{4}


seguidoras -pagadoras de regalías y dependientes de las decisiones tecnológicas extranjeras-, y para generar avances de punta para el mercado interno y la exportación, en el contexto de las reglas restrictivas de la OMC. ¿Qué hizo el Estado para lograr estos objetivos? En ambos casos, en lugar de desarrollar la tecnología en instituciones públicas (el modelo demiurgo de Evans, 1995), el Estado incitó la generación de redes de instituciones privadas (no sólo chabeols) y públicas para discutir y diseñar los proyectos, ${ }^{45}$ apoyó financieramente la investigación y desarrollo a través del MIC (los subsidios implicaban el monitoreo de los resultados y las empresas debían pagar a la larga la contribución financiera), protegió temporalmente los mercados procurando no contravenir las reglas de la OMC (hasta 2011, DMB era el único estándar de transmisión en el mercado coreano), favoreció la mejora tecnológica a partir de la experiencia en el mercado interno e impulsó la exportación de las nuevas tecnológica (para ello impulsó la aceptación internacional de los estándares tecnológicos desarrollados, aunque todavía no hay elementos claros para señalar el éxito externo de estos desarrollos tecnológicos). A fin de cuentas, con adaptaciones, el Estado coreano utilizó algunos de sus instrumentos favoritos (apoyo financiero, vigilancia de desempeño, protección de mercados y apoyos a la exportación), con medidas adaptadas a las circunstancias globales (Kim, 2012 A y B). Lejos está aún este modelo de una simple copia de las acciones de libre mercado y más cercano a una mezcla de los esquema de midwifery y husbandry de Evans (1995).

En breve, en el periodo de la crisis de 1997 y de dos presidencias progresistas o de centro izquierda, se manifestaron debilidades financieras severas de los conglomerados, además de presiones internas y externas para su 
reestructuración. Fue un periodo de exhibición de debilidades políticas de los conglomerados (interés del Estado en disminuir su poder, imagen de la opinión pública, críticas de organismos financieros internacionales); la entrada de la IED puede leerse también como la incorporación de un considerable competidor, que puede relativizar el poder de los conglomerados nacionales (fue claro esto en el sector bancario y en el automotriz). ${ }^{46}$ Sin embargo, los chaebols siguen siendo un jugador central en la economía coreana, han acumulado poder y relaciones internacionales que les permiten evadir algunas de las directivas estatales (Leem, 2000), generan tecnologías nuevas junto con otros actores locales (públicos y privados) y toman decisiones de manera autónoma; ${ }^{47}$ además, el manejo del supuesto del "too big to fail" sigue aportando poder de negociación a los conglomerados, como lo muestra la incorporación de políticas crediticias de apoyo en pleno siglo XXI. Podemos concluir que el Estado requiere de los chaebols, pero en situación de control -o de contención (Salas Porras, 2007)- y no con un poder desbocado; los chaebols buscan mayores márgenes de autonomía y cuentan con recursos para ello (López Aymes, 2005). No es la coordinación global de otras épocas (Shin y Chang, 2003) ni el custodio o demiurgo de Evans (1995), pero sí se trata aún de la reciprocidad de Amsden

46. Aunque López Aymes (2005, p. 213) en su trabajo de campo en Corea no encontró evidencia de utilización explícita de la IED como factor de debilitamiento de los conglomerados por parte del gobierno de Kim Dae Jung.

47. En 2004 y 2005, los gobiernos de Corea y México generaron un grupo bilateral de expertos para estudiar las posibilidades de un acuerdo de libre comercio. El gobierno mexicano pedía al coreano compromisos de inversión para poder dar el paso hacia un acuerdo de este tipo y en la última reunión del grupo, en la Secretaría de Economía mexicana en el Distrito Federal, fue invitado un alto directivo de Hyundai para saber las posibilidades de inversión de esta empresa en México. Flotaba la posibilidad de un anuncio espectacular para las delegaciones de Corea y México. La respuesta fue que las decisiones de inversión correspondían a la lógica de las necesidades corporativas globales de la empresa y que no había decisión específica para alguna nueva inversión en México (observación personal en esa reunión). 
aunque "gobernada" de acuerdo a Weiss (1998). La "nueva" interdependencia (Weiss, 1998) entre Estado y empresarios implica los apoyos a nuevos proyectos estratégicos (no necesariamente nuevas empresas), fundados por un conjunto de empresas privadas asociadas a instituciones públicas; sector público y sector privado comparten riesgos comunes, inversiones en ciencia y tecnología, y negociaciones internacionales delicadas (Evans, 1995).

El regreso de los conservadores: en busca del (imposible) regreso a la vieja relación (2008 en adelante)

En 2008 toma la presidencia un político en cierta manera soñado por los grandes conglomerados: Lee Myung Bak. Antes de ser alcalde de Seúl por el conservador Grand National Party, había sido director de la división de construcción de Hyundai. Después de dos intentos fallidos de algún representante de los chaebols para arribar a la presidencia (en 1992 y en 2003), lo logra Lee Myung Bak. Llegó a la presidencia con el proyecto de restauración de la vieja política económica centrada en el crecimiento -no en la "distribución" como sus dos antecesores progresistas- y de vinculación a los grandes empresarios a través de esquema de desregulación y privatización (reeditando tardíamente el discurso del Consenso de Washington, de los años noventa) (entrevista personal, 2008B). ${ }^{48}$

Desde sus primeras acciones, el presidente Lee se mostró como especialmente cercano a los conglomerados: generó incluso líneas directas de comunicación y eligió a cien empresarios para hablar directamente con ellos y man-

48. Nos dijo un analista político:"Pero Lee está en una especie de trampa: elevó las expectativas de los coreanos respecto del crecimiento; mucha gente dijo:"éste es el tipo'.Visto en perspectiva, la gente no necesariamente votó pro-Lee, sino anti-Roh, por la situación económica y por la firma del TLC. Los votos contra Roh se fueron hacia Lee, como votos de castigo". 
tenerse informado (The Hankyoreh, 2008); además anunció fuertes apoyos a los empresarios (planes masivos de infraestructura y apoyo a las industrias verdes, reconstrucción de cuatro ríos, construcción de nuevas plantas nucleares, reducciones fiscales para los grandes conglomerados) y eliminó en 2009 el sistema de topes de propiedad de empresas afiliadas a los chaebols (Kalinowski, 2009; Mukoyama, 2013). ${ }^{49}$ En este contexto, el peso económico de los chaebols continuó su progresión: las ventas de los diez conglomerados más grandes se incrementaron de representar 63.8\% del PIB en 2008 hasta 76.5\% en 2011 (Kwon, 2012).

Sin embargo, el presidente Lee no pudo llevar a cabo todas sus propuestas de privatizaciones debido, entre otros factores, a una inesperada protesta social en 2008 , a inicios del periodo de gobierno. ${ }^{50}$ Las fuertes movilizaciones de ese año iniciaron en contra de la autorización a la importación de carne bovina de los Estados Unidos - prohibida por cuestiones sanitarias en el anterior gobierno-, pero incluyeron otras demandas de manera creciente y entre ellas el freno a las privatizaciones anunciadas y el retiro del proyecto de conexión fluvial -gran canal- entre Seúl y Busán (Valencia, 2012).

El llamado hecho por Lee Myung Bak, en 2012, a los grandes conglomerados para retirarse de actividades manejadas tradicionalmente por pequeñas y medianas empresas (por ejemplo panaderías) muestra que el asunto chaebol sigue siendo un tema sensible y en el proceso electoral del segundo semestre de 2012 ocupó un lugar significativo en los debates entre los dos principales candidatos Park Geun Hye (Grand

49. Este sistema fue introducido en el gobierno de Chun Doo Hwan, en 1986, con un límite de $40 \%$ de los activos; después fue abolido en un corto periodo por Kim Dae Jung en 1998, para ser reinstalado en diciembre de 1999 con $25 \%$ de los activos; al final de la presidencia de Roh de nuevo fue reestablecido el límite de 40\% (Mukoyama, 20I3).

50. Logró la privatización de algunos aeropuertos y en 201 I anunció la privatización parcial y paulatina de la empresa ferroviaria KORAIL, que tiene la línea de alta velocidad entre Seúl y Pusán. 
National Party) y Moon Jai In (Democratic United Party): la "democratización económica", para el segundo con un fuerte control de los conglomerados (por ejemplo, disolución de inversiones cruzadas y reintroducción de los topes de inversión en empresa afiliadas) y para el primero con la prohibición de nuevas inversiones cruzadas. La ganadora Park Geun Hye ha indicado que la democratización económica implica el impulso de las pequeñas y medianas empresas al lado de los grandes conglomerados (Ahn, 2013). Semejante a lo que nos había expresado un miembro de un instituto de investigación económica de un gran conglomerado:

Hay un cambio en la gobernanza por los acuerdos en la OMC, que afecta la posibilidad de las políticas industriales. Ahora el gobierno subsidia a las PME y a los venture capitals en las PME, subsidia la agricultura y la inversión en infraestructura; apoya el desarrollo tecnológico de manera indirecta, no directa, a través de universidades. Las grandes compañías no necesitamos del gobierno para el desarrollo tecnológico porque ya tenemos nuestros propios centros de desarrollo tecnológico; si el gobierno interviniera directamente incluiría regulaciones y nosotros odiamos las regulaciones, no queremos estar en esta situación (entrevista personal, 2009).

\section{Conclusiones: las tensiones entre autonomía y asociación}

La relación Estado-empresarios en Corea está marcada por el inicio de las mismas en condiciones de desigualdad. Incluso puede decirse que, en esos inicios, una tarea del Estado fue la creación de la clase empresarial industrializadora (Woo-Cummings, 2000, p. 426). Esta creación se da en el contexto de experiencias excepcionales y difíciles para la sociedad (la Guerra de Corea), que requieren de medidas también excepcionales para consolidar los estados nacionales; el Estado optó por formas autoritarias de consolidación. 
No fue diferente la relación Estado-empresarios, también marcada por este autoritarismo inicial.

El creador es el fuerte de la relación, lo que genera al menos en un largo periodo la disposición entre los empresarios a acatar o seguir las directrices. Esta fortaleza no se da por generación espontánea, sino en el marco de las jerarquías sociales imperantes: el arresto de empresarios poderosos acusados de enriquecimiento ilícito en la Corea de los años sesenta. Con este punto de partida jerarquizado, Estado y empresarios van entrelazando acuerdos (instituciones) y conflictos; los empresarios van buscando cada vez mayor autonomía y el Estado va buscando, a su vez, mantener el control de la situación y definir reglas centrales (Weiss, 1998).

La reciprocidad incompleta, como institución o acuerdo implícito, es más clara en el caso coreano, especialmente en los años sesenta a ochenta. Los empresarios obtenían apoyos especiales en contrapartida al seguimiento de las líneas directrices -y en ocasiones mandatos explícitos- de las autoridades; los empresarios obtenían apoyos generalizados a través del proceso de control de las importaciones, aunque debían de competir por los apoyos para la promoción de las exportaciones. Sin embargo, las empresas que no seguían las líneas directrices de las políticas industriales podían ser "castigadas" de diversas formas, aunque la más importante era a través del control del crédito - tanto en la época de bancos en manos del sector público, como en el de la banca privatizada- y los apoyos especializados (incentivos fiscales).

El arma central que aseguraba la vigencia de la reciprocidad incompleta en Corea fue el control del crédito por parte del gobierno; la exposición al mercado externo funcionaba como un disciplinador más de las empresas que debían mostrar su eficiencia para seguir recibiendo apoyos fiscales y crediticios. En Corea se fue generando un 
importante proceso de empowerment del sector empresarial, especialmente de los grandes grupos corporativos apoyados por el Estado. Este crecimiento del poder empresarial se debió a diversos factores, entre los que resalta el enorme peso que fueron adquiriendo estos grupos en la economía coreana, en el mercado exportador y como productores en otros países. Esto permitió la generación de una institución informal sintetizada en el "too big to fail"; la suerte de la economía coreana se entrelazó cada vez más, al menos en la percepción pública, con la de los propios chaebols. Los conglomerados se fueron convirtiendo en factor infaltable en las negociaciones económicas y en varias ocasiones fueron salvados de quiebras o crisis notables.

Sin embargo, la autonomía lograda por estas empresas no les permitió lograr competir por la dirección moral-intelectual de la sociedad. El enorme poder económico y los logros indudables en el terreno tecnológico de estas empresas no fueron suficientes para superar el conflicto distributivo en la sociedad coreana. La desconfianza ciudadana -al menos en los periodos de crisis como 1987 y 1997- y la fortaleza histórica de los funcionarios coreanos (funcionarios de carrera, mayor estabilidad en las instituciones públicas), han impedido que esa autonomía se convierta en dominio en la relación entre Estado y conglomerados; aún entran en juego, en menor medida, elementos de la reciprocidad incompleta a principios del siglo XXI. Los acuerdos chaebols-Estado son actualmente vigilados cercanamente por organizaciones de la sociedad o por instituciones internacionales, y por la presencia de mayores intereses de empresas extranjeras en la economía coreana. La historia de la relación Estadoconglomerados en Corea no ha destruido la autonomía del sector público, pero tampoco ha generado empresas dependientes, sujetas siempre al apoyo público. Hasta ahora se ha tratado de una relación de mutua necesidad, 
con características de "interdependencia gobernada" que no terminan de desaparecer.

Fecha de recepción: 08 de enero de 2015 Fecha de aceptación: 28 de enero de 2015

Bibliografía

Ahn, Seon-Hee (20I3).“'In her inauguration, Park Geun-hye describes two-pronged approach of creative economy and economic democracy". The Hankyoreh. Recuperado de http://www.hani.co.kr/arti/english_edition/e_national/57557l.html

Amsden, Alice H. (1989). Asia's next giant. South Korea and Late Industrialisation. New York: Oxford University Press.

Bank of Korea (BOK) (1990). Financial System in Korea. Seúl: The Bank of Korea.

- (1998). Financial Crisis in Korea. Research Department. Seúl:The Bank of Korea.

Bello,Walden y Rosenfeld, S. (1990). Dragon in Distress.Asia's Miracle Economies in crisis. San Francisco: Institute for Food and Development Policy.

Boyer, Robert (1986). La théorie de la régulation: une analyse critique. París: Agalma-La Découverte.

Chang, Ha-Joon (2004).“The 1977 Korean Crisis: Causes and Consequences", en Edmund Amann y Ha-Joon Chang, Brazil and South Korea. Economic Crisis and Restructuring (pp. 107- 122). London: Institute of Latin American Studies, University of London.

Chang, S.W. (1993). The five-year Plan for the new economy (Summary). Seúl: EPB.

Choi, Jang-Jip (1983). Interest Conflict and Political Control in South Korea. Dissertation, Doctor of Philosophy. Chicago: The University of Chicago.

Chopra, Ajai et al. (2002). "From Crisis to Recovery in Korea: Strategy, Achivements and Lessons”, en David T. 
Coe y Se-jik Kim, Korean Crisis and Recovery (pp. I3-104). Bibliografía Washington: IMF and KIIEP.

Eckert, Carter J. (1993). "The South Korean Bourgeoisie: A Class in Search of Hegemony", en H. Koo, State and Society in Contemporary Korea (pp. 95-130). Ithaca: Cornell University Press.

Entrevista personal a funcionario del Banco de Corea (1992). Paris, Francia.

Entrevista personal a miembro del Comité Monetario (1993 A). Seúl, Corea del Sur.

Entrevista personal a representante sindical nacional (1993 B). Seúl, Corea del Sur.

Entrevista personal a académico (1999). Seúl, Corea del Sur. Entrevista personal a académico (2008 A). Seúl, Corea del Sur.

Entrevista personal a analista político (2008 B). Seúl, Corea del Sur.

Entrevista personal a miembro de instituto privado de investigación económica (2009). Seúl, Corea del Sur.

Evans, Peter (2008). In Search of The $2 I^{\text {st }}$ Century Developmental State. Working Paper 4. Reino Unido: The Centre for Global Political Economy, University of Sussex. (1995). Embedded Autonomy. States and Industrial Transformation. Princeton: Princeton University Press. Fields, Karl J. (1995). Entreprise and the State in Korea and Taiwan. Ithaca: Cornell University Press.

Haggard, Stephan and Chung-in Moon (1993). "The State, Politics, and Economic Development in Postwar South Korea", en H. Koo, State and Society in Contemporary Korea (pp. 5I-93). Ithaca: Cornell University Press.

Hart-Landesberg, Martin (1993). The Rush of Development. Economic Change and Political Struggle in South Korea. New York: Monthly Review Press.

Hayo, Bernd y Doh Shin (2002). Mass attitudes toward financial crisis and economic reform in Korea. SPP Working Paper 
363. Glasgow: University of Strathclyde, Centre for the Sudy of Public Policy.

Hira,Anil;james Morfopoulos y Florence Chee (2012).“Evolution of the South Korea Wireless Industry: from State Guidance to Global Competition". Internatonal Journal of Technology and Globalization, 6 (I/2), 65-86.

Jones, Leroy P. e II Sakong ( 1980). Government, Business, and Entrepreneurship in Economic Development: The Korean Case. Cambridge: Council of EastAsian Studies, Harvard University.

Kalinowski,Thomas (2009).“The Politics of Market Reforms: Korea's Path from Chaebol Republic to Market Democracy and Back". Contemporary Politics, 15 (3), 287-304. Kang, David C. (2002). Crony Capitalism. Corruption and Development in South Korea and the Philippines. Camdridge: University Press.

Kim, Kyeong-Won (2003). Post-Crisis Transformation of the Korean Economy.A Review from 1998 to 2002. Seúl:SERI. Kim, Sung-Young (2012A). “Transitioning from Fast-Follower to Innovator: The Institutional Foundations of the Korean Telecommunications Sector". Review of International Political Economy, 19 (I), I 40-I68.

(20I2B)."The Politics of Tchnological Upgrading in South Korea: How Government and Business Challenged the Might of Qualcomm". New Political Economy, I 7 (3), 293-3।2.

Kwark, E. K. Lawrencia (2000). Démocratisation et crise économique asiatique. Le cas de la Corée du Sud. Francia: Mimeo. Kwon Eun-Jung (28 de agosto de 20I2). "Big conglomerates expanding their control of an array of business areas". The Hankyoreh. Recuperado de http://www.hani.co.kr/ arti/english_edition/e_business/549028.html

Lanzarotti, Mario (1992). La Corée du Sud: une sortie du sous-développement. París: IEDES-PUF. 
Leem, Kuyng Hoon (marzo de 2000). Back to the future: the Bibliografía politics of economic reform under Kima Dae-Jung Presidency. Presentado en Conference "Korea: its international and comparative context". Harvard University.

Lim, G.C.(|99|).“'La transformación dinámica y el papel del gobierno: la lección de Corea y de los países de industrialización reciente", en O.Martínez Legorreta, Industria, comercio y Estado. Algunas experiencias en la Cuenca del Pacífico (pp. 335-352). México: Colegio de México.

Lim, Haeran (2010). "The Transformation of the Development State and Economic Reform in Korea". Journal of Contemporary Asia, 40 (2), 188-2 10.

Lipietz, Alain (1983). Le monde enchanté. De la valeur à l'envol inflationniste. París: La Découverte/Maspero.

López Aymes, Juan Felipe (2005). “La economía política del cambio institucional en Corea: inversión extranjera y relaciones gobierno-empresas", en Juan José Ramírez Bonilla (coord.), Transiciones coreanas. Permanencia y cambio en Corea del Sur en el inicio del siglo XXI (pp. I77219). México: El Colegio de México y Korea Foundation. Mako, William P. (2002). "Corporate Restructuring and Reform: Lessons from Korea”, en David T. Coe y Se-jik Kim, Korean Crisis and Recovery (pp. 203-228). Washington: IMF and KIIEPWashington.

Moon, Chung-In (199I). "The political economy of defense industrialization in South Korea: constraints, opportunities and prospects". The Journal of East Asia Affairs, 5 (2), été-automne 1991, 438-465.

Mukoyama, Hidehiko (20I3). "How will the Economic Policies of South Korea's New Administration Influence Economic Relations with Japan”. Pacific Business and Industries, 13 (47), pp. 2-24.

OECD (1999). OECD Economic Surveys: Korea 1999. París: OECD Publishing. (2003). OECD Economic Surveys. Korea. París: OECD. 
Bibliografía

Rennard, Jean Philippe (1993). Industrialisation tardive et nouvelles approches de l'économie mondiale. Le cas de l'industrie automobile en République de Corée. Grenoble: IREPD, Université Pierre Mendès France.

Sakong, II (1993). Korea in the World Economy. Washington: Institute for International Economic.

Salas-Porras, Alejandra (2007). "Los grupos mexicanos y coreanos ante la crisis del Estado". Foro Internacional, 47 (2), 300-339.

Sapir, Jacques (1989). L'économie mobilisée. París: La Découverte.

Seo, Ick-Jin (2000). La Corée du Sud. Una analyse historique du processus du développement. París: L'Harmattan.

Shim, Y. S. (1992). Les capitaux étrangers dans le processus d'industrialisation en Corée du Sud: interprétation à partir d'une logique endogène. Grenoble: Thèse, Université Pierre Mendès France.

Shin, Jang-Sup (2004)."Globalisation and Industrial Restructuring:The Case of South Korea", en Edmund Amann y Ha-Joon Chang, Brazil and South Korea. Economic Crisis and Restructuring (Pp. I23-I5 I). London: Institute of Latin American Studies, University of London.

Shin, Jang-Sup y Ha-Joon Chang (2003). Restructuring Korea Inc. Londres: RoutledgeCurzon.

Song, Byung-Nak (1990). The Rise of the Korean Economy. New York: Oxford University Press.

Steers, Richard M.; Yoo-Keun Shin y Gerardo R. Ungson (1989). The Chaebol. Korea's new industrial might. New York: Harper and Row.

Stiglitz, Joseph (2002). Globalization and its Discontents. New York: Norton.

The Hankyoreh (2008). "I 00 entrepreneurs picked for presidential hotline”. Recuperado de http://www.hani.co.kr/ arti/english_edition/e_business/276723.html 
Valencia Lomelí, Enrique (1996). Deux ajustements, deux traBibliografía jectories: les politiques d'ajustement de la Corée du Sud et du Mexiquie (1979-1992). Thèse de Doctorat en Sciences Economiques, Université de París VII, París.

(2012). "Coaliciones y transformación del régimen de bienestar en Corea del Sur (1987-20II)”, en José Luis Calva (coord.), Nueva estrategia de desarrollo (pp. 22I-25I). México: UNAM.

Wade, Roberto (1999). El mercado dirigido. La teoría económica y la función del gobierno en la industrialización del este de Asia. México: FCE.

Weiss, Linda (1998). The Myth of the Powerless State:Governing the Economy in a Global Era. Cambridge: Polity Press.

Woo, Meredith (199I). Race to the swift. State and Finance in Korean Industrialization. New York: Columbia University Press.

Woo-Cummings, Meredith (1999). The state and the reform of the corporate sector in Korea. Northwestern University: Mimeo.

(2000). "La economía política del crecimiento en el Asia Oriental. Una perspectiva del Estado, el mercado y la ideología”, en Masahiko Aoki, Hyung-Ki Kim y Masahiro Okuno-Fujiwara, El papel del gobierno en el desarrollo económico del Asdia Oriental. Análisis institucional comparado (pp. 4I4-437). México: FCE y Trimestre Económico.

Yi, Dae-Hoon Francis (1998). "Response of Korean civil society to the IMF's intervertion", en Pax RomanaICMICA, Seoul Forum on The Asian Economic Crisis and the Role of the Church.The IMF, Human Rights and the Church (pp. I27-I33). Seúl: Pax Romana-ICMICA.

Yoo, J. (1991). "La política de Corea para las industrias pesada y química en los años setenta: sus efectos en la productividad del capital y en la competitividad de las exportaciones", en O. Martínez Legorreta, Industria, 
Bibliografía comercio y Estado. Algunas experiencias en la Cuenca del Pacífico (pp. 257-290). México: Colegio de México.

You, J. y J. Lee (1999). Economic and social consequences of globalization:The case of South Korea. Seúl: Korea Development Institute, Mimeo. 